\title{
Neutral Hydrogen Gas in Star Forming Galaxies at $\mathrm{z}=0.24$
}

\author{
Philip Lah $^{1}$, Jayaram N. Chengalur ${ }^{2}$, Frank H. Briggs ${ }^{1,3}$, \\ Matthew Colless ${ }^{4}$, Roberto De Propris ${ }^{5}$, Michael B. Pracy ${ }^{1}$ \\ and W. J. G. de Blok ${ }^{1}$ \\ ${ }^{1}$ Research School of Astronomy \& Astrophysics, The Australian National University, Australia \\ ${ }^{2}$ National Centre for Radio Astrophysics, India \\ ${ }^{3}$ Australian Telescope National Facility, Australia \\ ${ }^{4}$ Anglo-Australian Observatory, Australia \\ ${ }^{5}$ Cerro Tololo Inter-American Observatory, Chile
}

\begin{abstract}
We use observations from the Giant Metrewave Radio Telescope (GMRT) to measure the atomic hydrogen gas content of star-forming galaxies at $\mathrm{z}=0.24$ (i.e. a look-backtime of $\sim 3$ Gyr). To measure the HI $21 \mathrm{~cm}$ emission signal we stack the signal from 121 galaxies with known optical positions and redshifts. We find an average HI mass for the galaxies of $(2.26 \pm 0.90) \times 10^{9} \mathrm{M}_{\odot}$. We translate this HI measurement into a cosmic density of neutral gas at $\mathrm{z}=0.24$ of $\Omega_{\mathrm{gas}}=(0.91 \pm 0.42) \times 10^{-3}$. This value is consistent with that estimated from damped Ly $\alpha$ systems around this redshift.
\end{abstract}

Keywords. galaxies: evolution, galaxies: ISM, radio lines: galaxies, radio continuum: galaxies

\section{Background}

The cosmic star formation rate density is known to increase by an order of magnitude between the present time and $\mathrm{z} \sim 1$. However, the cosmic density of neutral gas is poorly constrained over this redshift range. Consequently, we are missing a key element in our understanding of galaxy evolution, that of the fuel supply for new stars.

In the local universe $(\mathrm{z} \sim 0)$ the quantity of atomic hydrogen gas in galaxies has been measured with good precision using HI $21 \mathrm{~cm}$ emission surveys (Zwaan et al. 2005). However using radio observations of HI $21 \mathrm{~cm}$ emission to constrain the neutral gas density at cosmologically significant distances is difficult. This is because the flux from most individual galaxies at these redshifts falls below the detection limit of current radio telescopes in reasonable observing times.

Observations of damped Ly $\alpha$ absorption in QSO spectra have proven very effective at measuring the density of atomic hydrogen at high redshifts, $\mathrm{z}>1.5$ (Prochaska et al. 2005). However, it is difficult to constrain the neutral gas density from observations of damped Ly $\alpha$ absorbers at intermediate redshifts, since at these redshifts the Ly $\alpha$ line is not accessible by ground-based telescopes, and the average number of damped Ly $\alpha$ absorbers per unit redshift is low.

\section{Results}

We aim to measure the amount of neutral gas in galaxies at moderate redshifts using the HI $21 \mathrm{~cm}$ line by coadding the signal from multiple galaxies using their predetermined optical position and redshifts.

For this project we used the Giant Metrewave Radio Telescope (GMRT) to measure the atomic hydrogen gas content of star-forming galaxies at $\mathrm{z}=0.24$. Our sample of 


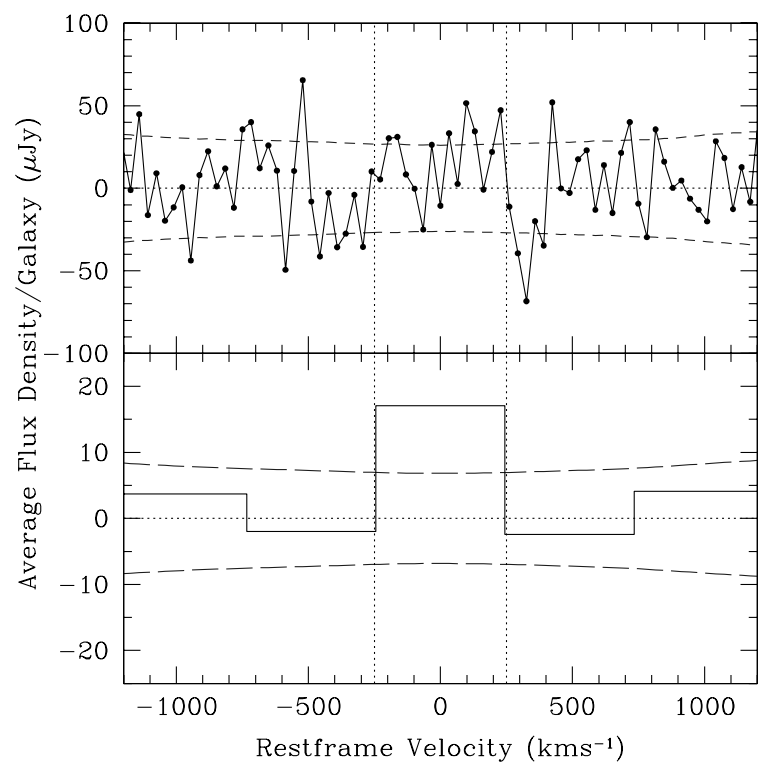

Figure 1. The average HI galaxy spectrum created from coadding the signal of all 121 galaxies with known optical redshifts. The top spectrum has no smoothing or binning and has a velocity step size of $32.6 \mathrm{~km} \mathrm{~s}^{-1}$. The bottom spectrum has been binned to $\sim 500 \mathrm{~km} \mathrm{~s}^{-1}$. For both spectra the $1 \sigma$ error is shown as dashed lines above and below zero.

galaxies was selected from $\mathrm{H} \alpha$-emitting field galaxies detected in a narrow-band imaging survey with the Subaru Telescope. The Anglo-Australian Telescope was used to obtain precise optical redshifts for these galaxies.

In $\sim 40$ hours of radio observations, we measure an average atomic hydrogen gas mass of $(2.26 \pm 0.90) \times 10^{9} \mathrm{M}_{\odot}$ from the coadded signal of 121 galaxies as seen in Figure 1. We translate this HI signal into a cosmic density of neutral gas at $\mathrm{z}=0.24$ of $\Omega_{\text {gas }}=(0.91 \pm 0.42) \times 10^{-3}$. This is the current highest redshift at which the neutral gas density has been constrained from $21 \mathrm{~cm}$ emission. Our value is consistent with that estimated from damped Ly $\alpha$ systems at the redshift of interest by Rao et al. (2006).

Using our data, we also find that the correlations between the $\mathrm{H} \alpha$ luminosity and the radio continuum luminosity and between the star formation rate and the HI gas content in star-forming galaxies at $\mathrm{z}=0.24$ are consistent with the correlations found at $\mathrm{z}=0$. These two results suggest that the star formation mechanisms in field galaxies $\sim 3$ Gyr ago were not substantially different from the present, even though the star formation rate is $\sim 3$ times higher.

For further information see Lah et al. (2007), MNRAS, 376, 1357.

\section{References}

Lah, P., et al. 2007, MNRAS, 376, 1357

Prochaska, J. X., Herbert-Fort, S., \& Wolfe, A. M. 2005, ApJ, 635, 123

Rao, S. M., Turnshek, D. A., \& Nestor, D. B. 2006, ApJ, 636, 610

Zwaan, M. A., Meyer, M. J., Staveley-Smith, L., \& Webster, R. L. 2005, MNRAS, 359, L30 